\title{
Wardrop Equilibrium Formulation of Resource-Constrained DTN Routing in Public Safety Networks
}

\author{
Pierre-Ugo Tournoux ${ }^{\star}$, Vania Conan ${ }^{\diamond}$, Jon Crowcroft ${ }^{\dagger}$, Jérémie Leguay ${ }^{\diamond}$, \\ Marcelo Dias de Amorim ${ }^{\ddagger}$, and Farid Benbadis $\diamond$ \\ ${ }^{\star}$ Université de Toulouse ${ }^{\diamond}$ Thales Communications ${ }^{\dagger}$ University of Cambridge ${ }^{\ddagger}$ UPMC Paris Universitas
}

\begin{abstract}
In this paper, we investigate the ability of using DTNs in Public Safety networks, where bandwidth and storage are constrained. We formalize the problem as a Wardrop equilibrium over a time-discretized graph. Driven by our findings, we propose RECOR, a centralized REsource-Constrained ORaclebased DTN routing mechanism, which spreads the demand across multiple store-carry-forward paths to satisfy the node storage and link transport constraints observed in intervention situations. By applying the proposed mechanism to real Bluetooth-based DTN traces, we show that the transmission bottleneck can be compensated, but only up to a certain extent, by increasing storage capacity and delay. We also analyze the benefit of strategies that provide more resources to highly connected nodes (e.g. ambulances and firetrucks) which can then feed incentives and policies for DTN network engineering. Finally, the idea presented here is general and suggests the necessity of tracedriven simulation and specific modeling tools for appropriate design of future DTN resource management policies.
\end{abstract}

Index Terms-DTN, Resource usage, Wardrop Equilibrium.

\section{INTRODUCTION}

One of the greatest challenges toward using DTN systems in Public Safety situations is to properly understand the impact of resource limitation on message routing. Most of the routing protocols proposed in the literature have been designed and compared under the assumption that storage and transmission capacities are unlimited and, more importantly, in the case of non-congested networks [1], [2].Although some papers have proposed routing schemes that take into account resource limitation at the nodes [3], [4], there is still a lack of clear and generic understanding of the impact of resource limitation, one of the characteristics of Public Safety interventions, on DTN performances. This question is fundamental as it is directly related to the practical feasibility of existing routing protocols in disaster situations, where public safety forces have to operate.

In this paper, we investigate the performance that can be expected in a DTN under finite storage and transmission constraints. Nodes decide to store, carry, or transfer messages independently of one another but based on the priority they give to storage, transmission, or delay. Nodes are ready to take part in routing messages of others as long as they can keep some control over the expected impact on their resources.
We formulate the problem above as a traffic assignment problem and investigate it using the first and second principles of equilibrium developed by John Glen Wardrop in 1952 [5]. Previous works have applied this idea to cellular, sensor, and ad hoc networks [6], [7], [8]. To the best of our knowledge, our work is the first to focus on DTNs and to generalize the analysis to both transmission and storage constraints. Wardrop principles, originally proposed for road traffic (but with much wider applicability), lead to the distribution of the load over the network minimizing resource usages. The first principle defines uncoordinated selfish resource usage whereas the second principle ensures minimal resource consumption. They lead respectively to the user equilibrium and the system optimum.

In this paper, we develop a methodology with the goal of investigating the performance of DTNs under Wardrop equilibria. Our contributions are threefold. Firstly, we express resource-constrained DTN routing as a Traffic Assignment Problem (TAP) over a Time-Discretized Graph (TDG) that captures the dynamic characteristics of the network. Based on the resulting Wardrop equilibrium, we propose RECOR, a centralized REsource-Constrained ORacle-based routing algorithm that offers a more realistic and comprehensive benchmark for analyzing the performance of practical routing schemes. RECOR considers not only delay but also network resource usage. Secondly, we analyze the interplay between node preferences and constraints, i.e., transmission cost, storage capacity, and acceptable delay. Node preferences (e.g., offering more storage or reducing delay constraints with the aim of increasing delivery ratio) are captured by corresponding cost functions associated to the edges of the time-discretized graph and yield different Wardrop equilibria. Finally, we investigate the benefits of providing increased capacity to popular nodes and assess the resulting performance gains. This provides an insight on how to provision future DTNs and design corresponding incentive strategies.

We apply our methodology to two scenarios - an ideal one for analysis purposes and a realistic one based on a real dataset. Our analyses address the major details of computational complexity, storage, and bounds on the global performance of the system. Our results reveal that: 


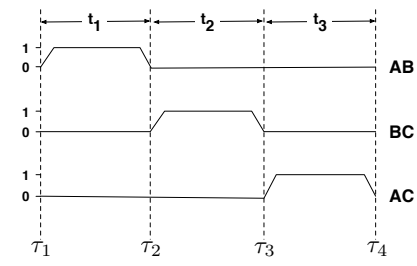

Fig. 1. Example of DTN contact patterns.

- Delay-tolerance helps increasing capacity,

- Infinite capacity might not help,

- Transmission-based incentives improve performance, and

- Routing protocols should be multi-dimensional.

\section{DTN AS A TIME-DISCRETIZED GRAPH}

We adopt a graph representation to model DTNs. Traditional DTN representation entails evolving graphs, where the vertices of the graph are wireless nodes and edges represent contact opportunities through time [9]. Even if such a representation is rich, it is difficult to manipulate. We propose to use a slightly different model, namely TDG (time-discretized graph). In a TDG, a path between two vertices represents a feasible spatiotemporal DTN route between two nodes. It is important to underline that this representation does not compromise the computation of classical problems such as shortest path [10] or other well known traffic assignment problems [11], [12], [13]. A time-discretized graph is similar in essence to timeindependent graphs proposed by Hay et al [14]. In both approaches, edges may be associated with different metrics to incorporate delay, storage, and transmission constraints. Nevertheless, time-independent graphs require the pruning of some contacts between nodes, which is not the case in TDG.

\section{A. TDG: Time-Discretized Graph Model}

We now show how to turn any DTN connectivity into a TDG through a simple example. Let us consider a DTN network composed of three nodes $A, B$, and $C$, as illustrated in Fig. 1 which shows the evolution of contacts between all pairs of nodes. For each possible contact $A B, A C$, and $B C$, the value one indicates that nodes are in radio range of each other and can communicate. We assume that contacts offer bidirectional connectivity. In the example, $A$ and $B$ are in contact during $t_{1}, B$ and $C$ are in contact during $t_{2}$, and $A$ and $C$ are in contact during $t_{3}$. Based on this configuration, if $A$ needs to transfer a message to $C$, it has the option to send it through $B$ or wait until it meets $C$. The choice is based on the result of the forwarding strategy.

To turn contact patterns between nodes from DTN connectivity into a TDG, we first discretize time into intervals of varying durations. Each time interval in TDG represents a period of time during which the topology of the network remains unchanged. The general idea is that vertices in a TDG represent the state of each node at each time step, and that the links between these vertices represent the store or forward opportunities between the nodes during the given time interval.

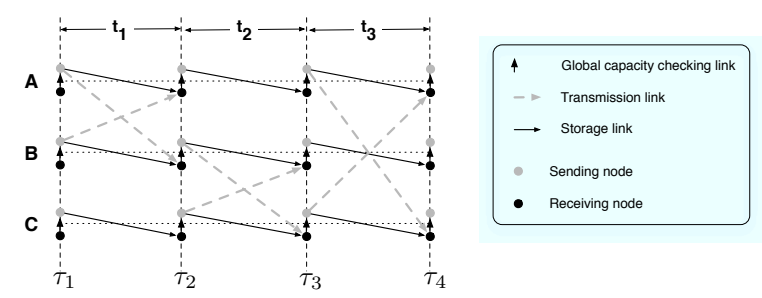

Fig. 2. The corresponding graph representation of the contact pattern shown in Fig. 1. Note that there are two different potential routes between $A$ and $C$, namely $A_{\tau_{1}}^{r} \overrightarrow{A_{\tau_{1}}^{r}} \rightarrow A_{\tau_{1}}^{s} \rightarrow A_{\tau_{2}}^{r} \rightarrow A_{\tau_{2}}^{s} \rightarrow A_{\tau_{3}}^{r} \rightarrow A_{\tau_{3}}^{s} \rightarrow C_{\tau_{4}}^{r}$ and

We also consider two state vertices for each node at each discreet time instant: sending and receiving. The idea is to decouple the reception of messages from their sending to have capacity constraint checking on storage capabilities (as explained below). For a given node $X$, we note these two states $X^{s}$ and $X^{r}$ for, respectively, sending and receiving.

A time-discretized graph $G=(V, E)$ represents DTN routes univoquely - any path in the graph represents a feasible DTN route, and, conversely, all feasible DTN routes can be represented as paths in the graph. ${ }^{1}$

Three types of links are used in Fig. 2, corresponding to the contact pattern of Fig. 1, each of them associated with three different steps in the communication process:

- Forwarding step. Illustrated by gray dashed arrows, the set $\mathcal{T}$ of forwarding edges form the set of forwarding represent symmetric contacts between two nodes and, consequently, transmission opportunities.

- Storage step. The set $\mathcal{S}$ of storage edges, shown as black solid arrows, correspond to the case where nodes keep data for one or more time intervals.

- Total storage constraint step. Shown as tiny vertical black arrows, the set $\mathcal{C}$ of edges are used for total storage constraint checking between receiving and sending states. These links are not related to any time duration, but occur for all nodes at all time instants. Such edges are need since the storage constraint applies to both stored and transmitted traffic - to transmit or receive data, we assume that nodes need to get the capacity to store data (which also explains the need for two states per node).

\section{B. Assigning costs to paths}

Every individual edge $e \in E$ of graph $G$ bears a cost $c(e)$. Each of the three types of edges have a different cost: (i) cost of radio transmission is associated with forwarding steps, (ii) cost of data storage is associated with storage steps, and (iii) total storage constraint checking is performed by a cost function that goes to infinity when the total data achieves the maximum allowed storage value. The total cost of each path is obtained by adding the individual costs of all traversed edges.

The use of different costs allows us to study congestion since costs become prohibitive on paths that have reached their

${ }^{1}$ For the sake of readability, we keep the traditional graph representation $G=(V, E)$. 
storage or transmission capacity limits. To handle routes with different total durations, a time value corresponding to the duration of the discretization interval is added to the costs above, both for the dashed and the plain edges since they correspond to steps of finite duration. Such a manipulation helps us analyze the impact of how nodes are tolerant to delay.

Note that further constraints could be added to the values of the data rates on each edge to model, for instance, interference in clustered environments, represented by nodes with high degree. The specific cost functions that we consider in this paper are presented in Section III-C.

\section{RECOR}

In this section, we propose RECOR, our REsourceConstrained ORacle based routing algorithm, which balances the network demand over multiple paths in order to satisfy network constraints and node storage capabilities. We assume that nodes are likely to behave in a selfish way, but not maliciously. The resulting system is a typical case of the vehicular traffic assignment problem. We investigate its steady state using the first principle of Wardrop [5], which we describe in the following section.

\section{A. General Wardrop equilibrium}

Recall that we use the time discretized graph to represent a DTN (as defined in Section II). Let $G=(V, E)$ be this graph, where $V$ represents the set of vertices in the network and $E$ the set of edges between pairs of vertices. An individual demand is expressed for an ordered pair of origin and destination nodes $(O, D)$ as the request to transfer $d$ bytes of data injected at time $\tau$. To allow DTN paths of different total durations, we add, for every node $N$, a particular vertex to the graph, namely $N_{\perp}$. All sending vertices $N_{\tau_{1}}^{s}, N_{\tau_{2}}^{s}, \ldots$ are linked to $N_{\perp}$ by an edge of null cost. Individual traffic demands can thus be expressed as the request to route $d$ bytes between the ordered pair $\left(O_{\tau}, N_{\perp}\right)$. This latter is renamed $(p, q)$ for the sake of simplicity.

The user equilibrium principle of Wardrop (aka the first principle of Wardrop [5]) states that for each origin-destination pair $(p, q)$ in set $\mathcal{D}$ of traffic demands, the utilized routes have equal and minimal total costs; no messages can decrease their total cost by shifting to another route for the given origindestination pair. Let us note $d_{p q}$ the fixed traffic demand sent by $p$ to $q$ and $R_{p q}$ the set of possible routes between $p$ and $q$. Each route $r \in R_{p q}$ is taken by the non-negative flow $h_{p q}^{r}$, where $c_{p q}^{r}$ is the non-negative travel cost on route $r$, and $\pi_{p q}$ the travel cost between $p$ and $q$ when the equilibrium is achieved (which does not depend on route $r$ ).

The equilibrium flow is defined by the following set of conditions:

- Condition 1. $\sum_{r \in R_{p q}} h_{p q}^{r}=d_{p q}$.

- Condition 2. $h_{p q}^{r}>0 \Rightarrow c_{p q}^{r}=\pi_{p q}, \forall r \in R_{p q}$.

- Condition 3. $h_{p q}^{r}=0 \Rightarrow c_{p q}^{r} \geq \pi_{p q}, \forall r \in R_{p q}$.

Condition 1 states that demand $d_{p, q}$ is split on $R_{p, q}$ routes which traffic $h_{p q}^{r}$ is not null. Condition 2 states that for every path $r$ used for demand $d_{p, q}$, traffic has the same cost $\pi_{p q}$, whatever followed path $r$. Finally, Condition 3 states that no traffic will travel on route $r$ if it increases the cost. In other words, every route which does not reduce the cost is eliminated. While the first condition expresses the flow distribution with respect to the fixed demands, the two first ones define the Wardrop principle. The equilibrium is interpreted as a Nash Equilibrium in a non-cooperative game among the $(p, q)$ pairs. It can be thought of as a steady state obtained after a transient phase in which origin nodes adjust the routes in order to minimize the cost of delivering demand $d_{p q}$.

Consider now the following hypothesis:

Hypothesis 1: There is at least one route for each demand $d_{p q}, d_{p q} \geq 0, \forall(p, q) \in \mathcal{D}$, given that all route cost functions $c_{p q}^{r}$ are positive and continuous (with respect to traffic load $h), \forall r \in R_{p q}$ are positive and continuous, and $(p, q) \in \mathcal{D}$.

For problems that satisfy Hypothesis 1, the Wardrop equilibrium is equivalent to the variational inequality problem of finding $h^{*}$ such that [15, pp. 83]:

$$
c\left(h^{*}\right)^{T}\left(h-h^{*}\right) \geq 0 .
$$

Note that the properties that are necessary for Hypothesis 1 to hold are very general. The cost functions do not have to be separable - the cost of an edge does not have to depend on the traffic along the edge only, but can depend on neighbor nodes for example. The cost functions do not need to be additive either, i.e., they do not need to be obtained as the sum of the individual costs of edges on the route (although we will make this assumption below for the sake of simplification). This means that one can also consider the complex behavior of the radio medium in the model.

\section{B. Simplifications and Implementation}

We introduce some simplifying hypothesis that lead to more stable and predictable results. First, we consider that the cost of paths on the time-discretized graph is defined as the sum of the costs of the traversed edges (the total cost is thus additive). Second, we assume that edge costs are separable. This amounts to neglecting some of the features of the radio medium. When considering a wireless transmission between two nodes, we consider that traffic can be split in both directions with an equal split of maximum bandwidth limit in each direction.Under these complementary hypotheses of additive separable costs, the variational Equation 1 can be expressed as a convex optimization problem defined as follows [16], [17], [18]:

$$
\min \operatorname{Cost}(f)=\sum_{e \in E} \int_{0}^{f_{e}} c_{e}(s) d s,
$$

subject to:

$$
\sum_{r \in R_{p q}} h_{p q}^{r}=d_{p q}, \forall(p, q) \in \mathcal{D}
$$

and 


$$
\sum_{(p, q) \in \mathcal{D}} \sum_{r \in R_{p q}} \delta_{p q}^{r e} h_{p q}^{r}=f_{e}, \forall e \in E
$$

where $\delta_{p q}^{r e}=1$ if route $r \in R_{p q}$ uses edge $e$ and 0 otherwise (it is the edge-route incidence matrix), and $f_{e}$ is the total flow on edge $e$.

This is an instance of the traffic assignment problem [11], [12], [13]. To resolve it, we use the Frank-Wolfe algorithm designed to solve quadratic programming problems with linear constraints [19]. The problem is to minimize $f(x)$ subject to $x \in S$, where $S \subset \mathbf{R}^{n}$ is a polyhedron and $f: \mathbf{R}^{n} \mapsto \mathbf{R}^{n}$ is a continuously differentiable function. For a detailed description of the algorithm, refer to Patriksson [15]. It alternates between the solution of a linear program defined by a tangential approximation of the objective and a line search minimizing the original objective over the line segment defined by the current iterate and the solution to the linear program.

\section{Cost Model for Resources}

To fully specify our instance of the traffic assignment problem, we further define practical cost functions that model the DTN system resources and integrate the user's willingness to use them. The cost functions are defined for every edge $e$. They make use of two types of parameters. The first type models the nodes' actual capabilities and the second one models the users' preferences.

Node capabilities are defined as follows. $C_{T}(e)$ is the maximum transmission capacity of edge $e \in \mathcal{T}$ and $C_{S}(e)$ is the maximum storage capacity of edge $e \in \mathcal{C}$. Large values of $w_{\Delta}$ lead to Wardrop equilibria with short delays, while routes with longer delays are penalized by additional $\Delta w_{\Delta}$ costs. As $w_{\Delta}$ tunes the delay tolerance of the user, $w_{\Delta}=0$ represents full delay tolerance and very large values $\left(w_{\Delta} \gg 1\right)$ would entail the use of shortest paths in terms of delay. For a given delay tolerance $w_{\Delta}$, which we analyze in Section IV, small values of $w_{T}\left(w_{T} \rightarrow 0\right)$ would favor DTN routes with multiple storage steps (and few transmission steps); on the other hand, large values of $w_{T}\left(w_{T} \gg 1\right)$ would favor DTN routes with several transmission steps.

Although we introduce three types of criteria (delay tolerance, storage, and transmission preferences), there are only two independent parameters since the storage criterion is scaleindependent.

For the definition of the cost functions, let us first introduce the following extra notations for our model: (i) the time step $\Delta$, (ii) $f(e)$, i.e., the function that returns the total number of flows assigned to link $e$, and (iii) the sharpness parameter $p$, which defines how fast the function curves toward the capacity limits. The choice of $p$ is an implementation issue; a large $p$ leads to more accuracy but generates higher cost values which may exceed the memory fields of program variables (i.e., int, float, double) in case of large networks.

The cost functions for the three different types of edges are thus defined by:

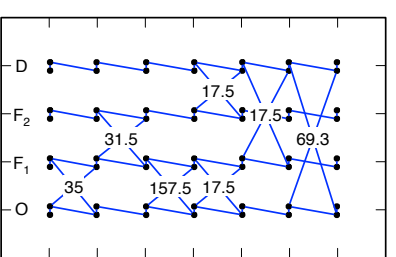

(a) Empty graph with link transmission capacity.

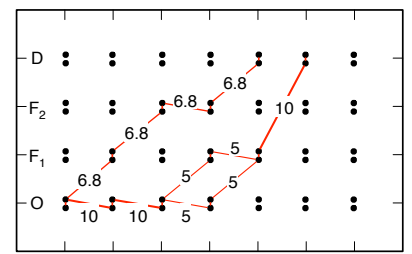

(c) Delay cost $\times 1.0$ and transmission cost $\times 30.0$.

sion cost $\times 30.0$

Fig. 3. Illustrative example of a 4-node topology. In this figure, the time step $\Delta$ is set to $225 \mathrm{~s}$, buffer size $C_{s}$ to $50 \mathrm{MB}$, while transmission capacity varies. Each link transmission capacity, in MB, depends on the contact duration and is represented, in Fig. 3(a), as a label on the corresponding link. The traffic demand $c$ from $O$ to $D$ is $16.8 \mathrm{MB}$ (24s $\times 700 \mathrm{Kbps})$. Fig. 3(a), 3(b), and 3(c) depict how this demand is balanced over the network depending on variable delay and transmission costs.

$$
\begin{gathered}
C(e \in \mathcal{T})=\Delta \cdot w_{\Delta}+w_{T} \cdot\left(f(e)+\frac{f(e)^{p}}{\left(C_{T}(e)\right)^{p-1}}\right), \\
C(e \in \mathcal{S})=\Delta \cdot w_{\Delta}, \\
C(e \in \mathcal{C})=\frac{f(e)^{p}}{\left(C_{S}(e)\right)^{p-1}} .
\end{gathered}
$$

\section{IMPACT OF RECOR PARAMETERS}

Let us now provide a first analysis of the interplay of the different parameters of the model. In the first example, we compare how RECOR spreads traffic demands for different values of the user preference parameters. Then, by using a real DTN dataset, we investigate the influence of delay tolerance on the performance of the system.

\section{A. A 4-node DTN example}

The purpose of this section is to explain, through a practical example, how user preferences impact the way Wardrop spreads traffic among storage and transmission links. Traffic assignment is done according to cost functions defined in Section III-C.

Fig. 5 gives a graphical insight into how the parameters impact the traffic assignment and network usage. Fig. 3(a) describes a basic network of four nodes $O, F_{1}, F_{2}$, and $D$. Node $O$ is the origin of the demand and $D$ its destination. Time is discretized into six time intervals of length $\Delta=225 \mathrm{~s}$. Note that there are five different loop-free paths between $O$ and $D$. The shortest delay that can be obtained is $4 \times \Delta$, and the smallest number of transmissions is 1 , if $O$ waits for 6 
time intervals until it meets $D$. The transmission capacities are noted on the corresponding links in Fig. 3(a) and buffers capacities are equal for all nodes and fixed to $50 \mathrm{MB}$. The traffic demand is $700 \mathrm{Kbps}$ during $24 \mathrm{~s}$ (total $=16.8 \mathrm{MB}$ ).

The remaining subfigures show three different traffic assignment configurations, as outputs of different sets of user requirements. We only show the links that transport a share of the traffic, their thickness is proportional to the share, which value, in megabytes, is labeled on the link. Each figure is the result of a specific priority in the traffic assignment algorithm:

Real-time requirements. When nodes are intolerant to delay, $w_{T}$ is set to a small value. Fig. 3(b) illustrates this case for $w_{T}=1$ and $w_{\Delta}=10$. Note that only one route is used in this case.

Scarce and expensive transmissions. This case is shown in Fig. 3(c). The real-time requirement is the same of the previous case but transmissions are also considered very costly. We set $w_{T}=30$, which drastically increases the linear cost of transmission links. This results in some more load balancing through the use of two more paths of longer delay and smaller number of hops.

Full delay-tolerance and expensive transmissions. This case corresponds to the other extreme, i.e., nodes are fully tolerant to delay but face expensive transmission links. We set $w_{\Delta}=0$ and $w_{T}=30$ (as in the previous case) and show the resulting configuration in Fig. 3(d). The interesting point here is that more paths are explored, although most of the traffic is sent through the direct link between $O$ and $D$.

For the sake of simplicity and comprehension, we have shown here an example with a single traffic demand between a pair of nodes. In a real DTN with several nodes and demands, there might have some unexpected behaviors due to the mutual influence of the different traffics. For this reason, we evaluate in the following our methodology on a dataset collected in a real DTN scenario.

\section{B. DTN dataset}

We use real connectivity data from the RollerNet dataset [20]. This dataset consists of proximity contacts between participants of the rollerblading tour in Paris using iMotes (Bluetooth contact loggers from Intel). This dataset shows high intensity of interactions between mobile nodes where the number of connectivity disruptions prevent the use of classical MANET protocols. We considered a portion of 2,000 s and selected 40 skaters.

\section{Tolerance to delay}

We focus on $w_{\Delta}$ (recall, the parameter in the cost functions that controls delay-tolerance). We evaluate on the RollerNet dataset how $w_{\Delta}$ influences the end-to-end transmission delay of traffic demands. Indeed, increasing $w_{\Delta}$ reduces delaytolerance of demands and forces traffic assignment to use shorter paths in terms of delay (as shown in the example of Section IV-A). To build the TDG graph out of the RollerNet

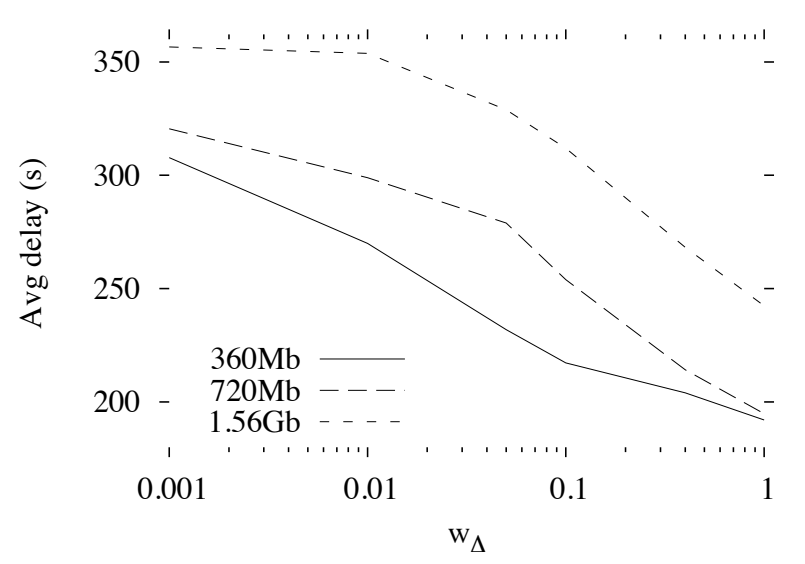

Fig. 4. Impact of $w_{\Delta}$ (log-scale) on the average delay. The three curves correspond to different input load (360 MB, $720 \mathrm{MB}$, and 1.56 GB).

dataset, we chose $\Delta=100 \mathrm{~s}, w_{T}=1$, and $w_{S}=1$. We assigned traffic flows between 15 pairs of nodes and generate data at times $t=0 \mathrm{~s}, t=600 \mathrm{~s}$ and $t=1,200 \mathrm{~s}$.

Fig. 4 shows the impact of $w_{\Delta}$ on the end-to-end transmission delay. It plots the delay averaged for all the demand as a function of $w_{\Delta}$. The three different curves are related to a load of respectively $360 \mathrm{MB}, 720 \mathrm{MB}$, and $1.56 \mathrm{~GB}$. For $w_{\Delta}=0.001$, the average delays are respectively $307.75 \mathrm{~s}$, $320.48 \mathrm{~s}$, and 356.63s. This figure shows that, as expected, increasing the load leads to an increase of transmission delay. On the other hand, the delay decreases inversely to $w_{\Delta}$ and independently of the load. This is due to the fact that delay constraints prevent the use of time consuming paths, thus reducing the average delay.

\section{DTN ENGINEERING}

As DTN nodes can be selfish in the way they utilize transmission and storage resources, incentive mechanisms have to be proposed. These mechanisms can encourage for instance bottleneck users to allocate more resources for the others and increase overall performance. In this context, we show how RECOR can be used to estimate the benefits of a connectivitybased resource allocation policy, both in terms of system costs and network capacity.

\section{A. Resource allocation policy}

We define a simple connectivity-based resource allocation policy that allocates radio bandwidth to nodes proportionally to their data forwarding capabilities. In other words, we define a policy that allocates more resources to nodes meeting more frequently other nodes and with longer contact times. We assume that rewards can be distributed to the nodes, so that they accept such allocation policy (e.g., offering free hardware upgrade, vouchers, or reductions on cellular phone calls).

We build this allocation policy upon a metric that calculates the amount of transfer opportunities that nodes have with others. To discriminate between nodes that meet a large 
number of nodes and those that meet some nodes but with long contact durations, we define the metric $m_{i}$ for node $i$ as:

$$
m_{i}=\frac{\sum_{t+\delta}^{N} d_{\delta}^{i}(t) \times l_{\delta}^{i}(t)}{N-\delta},
$$

where $d_{\delta}^{i}(t)$ is the amount of nodes encountered by node $i$ during the latest $\delta$ seconds and $l_{\delta}^{i}(t)$ is the sum of all these contact durations during the same $\delta$ seconds. For each node, this value is computed every second and the metric $m_{i}$ averages this product over a period of length $N$ seconds.

To obtain the final metric $w_{i}$ for a given node $i$, we calculate the ratio between $m_{i}$ and its average value for all nodes. This allows us to maintain an average value $m_{i}$ equal to 1 , thus facilitating the comparison between this allocation scheme and a uniform allocation policy. Indeed, the outgoing bandwidth allocated to nodes is obtained by the product Average bandwidth $\times w_{i}$. The total capacity in the network remains the same for the uniform and connectivity-based allocation schemes.

\section{B. Evaluation results}

We use the same parameters as in Section IV-C to build the TDG graph, i.e. $\Delta=100 \mathrm{~s}, w_{T}=1$, and $w_{S}=1$, and choose $w_{\Delta}=10^{-3}$ (we consider that flows are not delay constrained). We also assign traffic flows between 15 pairs of nodes and generate data at times $t=0 \mathrm{~s}, t=600 \mathrm{~s}$, and $t=1,200 \mathrm{~s}$. We run the Wardrop traffic assignment strategy 28 times, increasing the input traffic load from 100 KBytes to 40 MBytes. The time window used to estimate connectivity of nodes in the resource allocation policy is of size $\delta$ equal to 30 seconds.

To evaluate the performance of the connectivity-based resource allocation (denoted "Connectivity"), we compare this approach to a uniform resource assignment where nodes all have the same capacity (denoted "Equal"). We can see in Fig. 5(a) that, despite the global resources in the network remain the same for both policies, the routing cost induced is significantly lower when the radio resources are allocated function of nodes data forwarding capabilities. This exhibits the fact that the resources are more efficiently used.

Fig. 5(b) shows the total amount of load that is assigned over the capacity of the links as a function the total load input in the network. Note that the use of the metric significantly reduces the global link overload. We consider that the capacity of the network is reached when the global link overload increases linearly. Following this definition, we estimate that the capacity of the network accepts a maximum input load of 650 MBytes for Uniform and approximatively 900 MBytes for Connectivity. Such resource allocation also allows to reduce the delay which is in average of 323.3 seconds for Uniform and 316 seconds for Connectivity.

Whereas these results have demonstrated the use of RECOR for the evaluation of a simple resource allocation policy, RECOR can be used in the engineering of much more complex strategies and policies. For instance, it can be used to study dynamic resource allocation over time or to analyze

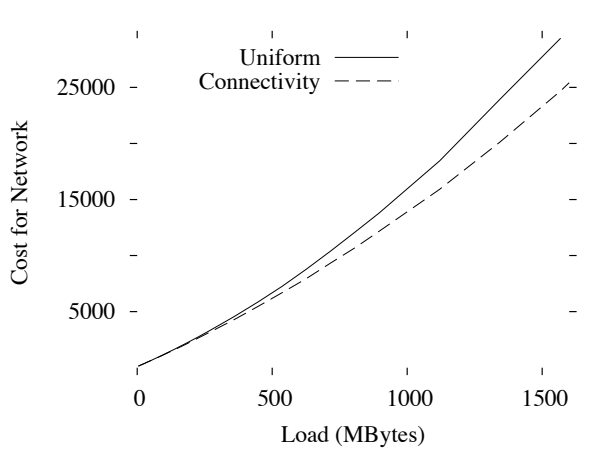

(a) Global cost vs. input load for each resource allocation policy.

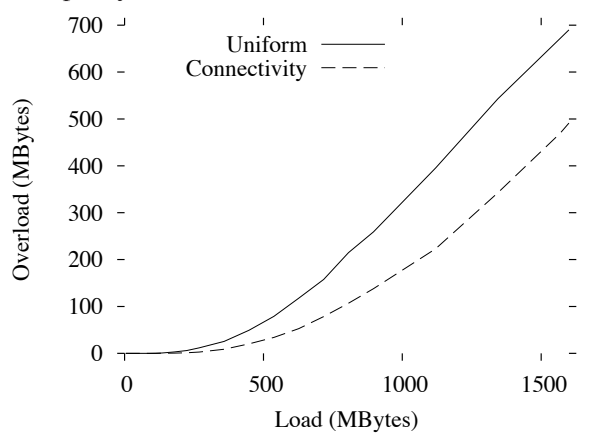

(b) Global link overload vs. input for each resource allocation policy.

Fig. 5.

the consequences of non-cooperative behaviors considering unidirectional connectivity for some nodes.

\section{RELATED WORK}

This paper also builds on the extensive literature on Wardrop equilibrium originally coming from road planning. It remains at the present time the most realistic model of congestion build up in road networks and the most common used model for road planning [23].

In 1956, Beckmann showed how to express the Wardrop's principles as a convex optimization problem and proposed algorithms to compute the equilibrium [16]. Other researchers have expanded the mathematical framework, introducing in particular its variational version, and enriched the algorithmic toolbox, as reviewed by Patriksson [15].

In order to study the impact of resource constraints on DTN performance, previous work in the domain have adopted empirical approaches using performance evaluation of practical routing strategies. For example, PRoPHET relies on a probabilistic metric calculated using history of encounters and transitivity [2]. Burgess et al. propose MaxProp in the context of DieselNet, a real DTN deployment on 40 buses [24], [25]. This protocol uses meeting probabilities to find paths in association to complementary mechanisms for improving performance in terms of delivery ratio and latency such as buffer management and transmission scheduling. Finally, Balasubramanian et al. have shown that routing strategies should 
fully integrate those parameters when making their forwarding decisions [26].

Our work definitely adds to this domain by providing a comprehensive formal approach that captures both resource constraints and the nodes' preferences and behavior.

\section{CONCLUSION}

We have addressed the problem of analyzing the impact of storage and transmission limitations on DTN message routing, where users have selfish behavior, because of their constant need of communication resources, and can express various preferences over the amount of resources they are ready to devote to DTN routing and priorities they can set, in particular for delay. The main contribution of this paper is a comprehensive formalization of this problem based on the first principle of Wardrop. Run on the time-discretized graph (TDG), a graph representation of the DTN dynamics, the corresponding transport assignment problem defines RECOR, an Oracle based routing algorithm which provides a tool to understand the impact of network resource usage on the DTN performances, independently of the specific routing protocol.

We applied our methodology to two scenarios: one theoretical and one realistic. We reached the conclusions that network capacity increases when users are more tolerant to delay and that adding resources to the network does not automatically lead to better performance. We also show the benefit of providing increased capacity to popular nodes and assess the performance gains this induces, thus paving the way for designing incentive strategies for improving DTN routing performances in public safety usage.

We believe that our work helps design, analyze, and enhance future DTN routing protocols and resource management policies, provinding a new way for public safety communications. Future work includes exploring the behavior of different controlled epidemic routing strategy against the RECOR bounds. We also believe that our methodology has wider implications than the ones explored in this paper. In particular applying such an approach to classes of randomized DTN graphs would offer a complementary structural point of view.

\section{REFERENCES}

[1] J. Leguay, T. Friedman, and V. Conan, "Evaluating mobility pattern space routing for DTNs," in Proc. INFOCOM, 2006.

[2] A. Lindgren, A. Doria, and O. Schelen, "Probabilistic routing in intermittently connected networks," in Proc. SAPIR, 2004.

[3] B. Burns, O. Brock, and B. N. Levine, "MV routing and capacity building in disruption tolerant networks," in Proc. Infocom, 2005.

[4] H Juang, H. Oki, Y. Wang, M. Martonosi, L. Peh, and D. Rubenstein, "Energy-efficient computing for wildlife tracking: Design tradeoffs and early experiences with Zebranet," in Proc. ASPLOS-X, 2002.

[5] J. Wardrop, "Some theoretical aspects of road traffic research," Proceedings of the Institution of Civil Engineers, Part II, vol. 1, no. 36, pp. 352-362, 1952.

[6] N. Bonneau, M. Debbah, E. Altman, and A. Hjørungnes, "Wardrop equilibrium for cdma systems," in IEEE WiOpt Workshop on Resource Allocation in Wireless Networks, Limassol, Cyprus, Apr. 2007.

[7] M. Munir, A. Kherani, and F. Filali, "A distributed algorithm to achieve cesaro-wardrop equilibrium in wireless sensor networks," in IEEE Consumer Communications and Networking Conference, Las Vegas, USA, Jan. 2008.
[8] V. Raghunathan and P. R. Kumar, "Issues in wardrop routing in wireless networks," in IEEE International Conference on Wireless Internet, Budapest, Hungary, July 2005.

[9] A. Ferreira, "Building a reference combinatorial model for manets," IEEE Network, vol. 18, no. 5, pp. 24-29, 2004.

[10] F. Harari, Graph Theory, Addison-Wesley, Reading, MA, 1969.

[11] H. Bar-Gera, "Origin-based algorithm for traffic assignment problem," Transportation Science, vol. 36, no. 4, pp. 398-417, 2002.

[12] E.M. Gafni and D.P. Bertsekas, "Two-metric projection methods for constrained optimization," SIAM Journal on Control and Optimization, vol. 22, no. 6, pp. 936-964, 1984

[13] A. Ouorou, P. Mahey, and J.-P. Vial, "A survey of algorithms for convex multicommodity flow problems," Management Science, vol. 46, pp. 126147,2000

[14] David Hay and Paolo Giaccone, "Optimal routing and scheduling for deterministic delay tolerant networks," in WONS'09: Proceedings of the Sixth international conference on Wireless On-Demand Network Systems and Services, Piscataway, NJ, USA, 2009, pp. 25-32, IEEE Press.

[15] M. Patriksson, The Traffic Assignment Problem: Models and Methods, VSP, Utrecht, 1994

[16] M. Beckmann, C. B. MCGUIRE, and C. B. ANDWINSTEN, Studies in the Economics of Transportation, Yale University Press, 1956.

[17] S. Dafermos, "The traffic assignment problem for multiclass-user transportation networks," Transportation Science, , no. 6, pp. 73-87, 1972.

[18] Torbjrn Larsson and Michael Patriksson, "Simplicial decomposition with disaggregated representation for the traffic assignment problem," Transportation Science, , no. 26, pp. 4-17, 1992.

[19] M. Frank and P. Wolfe, "An algorithm for quadratic programming," Naval Research Logistics Quarterly, , no. 3, pp. 95-110, 1956.

[20] P.-U. Tournoux, J. Leguay, F. Benbadis, V. Conan, M.D. de Amorim, and J. Whitbeck, "The accordion phenomenon: Analysis, characterization, and impact on dtn routing," in INFOCOM 2009, IEEE, April 2009, pp. $1116-1124$

[21] Tim Roughgarden and va Tardos, "How bad is selfish routing," Journal of the ACM, vol. 49, pp. 236-259, 2002.

[22] T. Larsson and M. Patriksson, "Side constrained traffic equilibrium models-analysis, computation and applications," Transportation Research, vol. 33B, pp. 233-264, 1999.

[23] Y. Sheffi, Urban Transportation Networks: Equilibrium Analysis with Mathematical Programming Methods, Prentice-Hall, Inc., 1985.

[24] J. Burgess, B. Gallagher, D. Jensen, and B. N. Levine, "Maxprop: Routing for vehicle-based disruption-tolerant networks," in IEEE Infocom, Barcelona, Spain, Apr. 2006.

[25] UMassDieselNet, "A Bus-based Disruption Tolerant Network," http: //prisms.cs.umass.edu/diesel/.

[26] A. Balasubramanian, B. Levine, and A. Venkataramani, "Dtn routing as a resource allocation problem," in ACM Sigcomm 2007, Kyoto, Japan, Aug. 2007. 